\section{ACIDENTES DE TRABALHO GRAVES ENVOLVENDO CRIANÇAS E ADOLESCENTES NA BAHIA, NO PERÍODO DE 2010 A 2018}

\section{SERIOUS WORK ACCIDENTS INVOLVING CHILDREN AND ADOLESCENTS IN BAHIA FROM 2010 TO 2018}

\section{Beatriz de Almeida Marques ${ }^{1}$ / Grasielle da Silva Santos ${ }^{1}$ / Iara Caroline Moura Conceição da Silva ${ }^{1} /$ Marcela Andrade Rios ${ }^{1} /$ Raissa Neyla da Silva Domingues Nogueira ${ }^{1}$ / Wesley dos Santos Teixeira' ${ }^{1, *}$}

\section{INTRODUÇÃO}

O trabalho infanto-juvenil é uma temática de grande relevância social e envolve inúmeras características tais como culturais, econômicas, psicológicas, físicas, dentre outras. Esse tipo de trabalho tem despertado a atenção da sociedade para a exploração da infância e juventude de milhares de crianças e adolescentes, cuja solução se baseia na erradicação necessária dessa prática, principalmente quando o trabalho aos quais mesmos estão submetidos envolve a execução de atividades degradantes e insalubres (PIMENTA et al., 2013).

Os acidentes de trabalho (AT) destacam-se como um grave problema de saúde, pois afetam um grande número de trabalhadores, entre eles os jovens considerados em idade produtiva. Esses eventos ocorridos no exercício do trabalho independem da situação empregatícia e previdenciária ao qual o trabalhador acidentado está submetido, ocasionando, assim, danos à saúde, ocasionado lesão corporal ou disfunção funcional que leve direta ou indiretamente ao óbito ou a perda permanente ou temporária da funcionalidade para atividade laboral (BRASIL, 2006).

\section{RESUMO}

Objetivo: Descrever o perfil dos casos de acidentes de trabalho graves em crianças e adolescentes, ocorridos entre 2010 a 2018, no estado da Bahia. Métodos: Estudo descritivo baseado em dados secundários extraídos do Sistema de Informações de Agravos de Notificação, acerca dos casos de acidentes de trabalho grave em crianças e adolescentes, ocorridos de 2010 a 2018, na Bahia. Para seleção dos acidentes de trabalho foi utilizado o código da CID 10 Y 96. Os dados foram tabulados e analisados através do Microsoft Office Excel 2010. Por se tratar de um estudo com base em dados secundário não houve submissão ao Comitê de Ética em Pesquisa. Resultados: O maior registro de notificação foi no ano de $2018(13,8 \%)$, na macrorregião leste $(32,3 \%)$, sexo masculino (90,7\%), na faixa etária entre 15 e 19 anos $(76,2 \%)$, raça $\neg /$ cor parda $(51,3 \%)$. A maior parte das vítimas estava nas instalações dos contratantes $(39,4 \%)$, sofreram acidentes típicos $(69,9 \%)$, a maior parte obteve um regime de tratamento hospitalar $(60,8 \%)$, evoluiu com incapacidade temporária causada pelo acidente $(33,4 \%)$ e $2,1 \%$ vieram a óbito. Conclusão: Evidencia-se a importância das esferas governamentais atuarem no desenvolvimento de políticas públicas que combatam o trabalho infantil, bem como promovam ambientes saudáveis de trabalho para jovens aprendizes.

Palavras-chave: Adolescente. Trabalho infantil. Acidentes de trabalho.

\section{ABSTRACT}

Objective: To describe the profile of cases of serious work accidents in children and adolescents, which occurred between 2010 and 2018, in the state of Bahia.Methods: Descriptive study based on secondary data extracted from the Notifiable Diseases Information System, about cases of serious work accidents in children and adolescents, which occurred from 2010 to 2018 in Bahia. The Y 96 code was used to select occupational accidents. The data were tabulated and analyzed using Microsoft Office Excel 2010. As this is a study based on secondary data, there was no submission to the Research Ethics Committee. Results: The highest record of notification was in $2018(13.8 \%)$, in the eastern macro-region $(32.3 \%)$, male $(90.7 \%)$, aged between 15 and 19 years $(76.2 \%)$, brown race $(51.3 \%)$. Most of the victims were in the contracting facilities (39.4\%), suffered typical accidents $(69.9 \%)$, most obtained a hospital treatment regime $(60.8 \%)$, evolved with temporary disability caused by the accident ( $33.4 \%$ ) and $2.1 \%$ died. Conclusion: It is evident the importance of governmental spheres to act in the development of public policies that combat child labor, as well as promoting healthy work environments for young apprentices.

Keywords: Adolescent. Child Labor. Accidents. Occupational.

Submetido em: 08 de nov. 2019

Aceito em: 14 de fev. 2020

${ }^{1}$ Universidade do Estado da Bahia - UNEB, Guanambi, Bahia - Brasil.

E-mail para correspondência: dossantosteixeirawesley@gmail.com

Rev. ComCiência - jun. 2020, vol. 5, no. 6, p. 53-57/ doi: 10.36112/ issn2595-1890.v5.i6.p53-57 
Neste contexto de riscos no processo de trabalho e prejuízos ao indivíduo, o combate contra o trabalho infanto-juvenil vem sendo realizado cada vez mais cedo com o intuito de inibir a atividade laboral precoce. Esta luta ocupa um lugar de destaque na conjuntura política pública mundial, pois conseguiu maior atenção dos representantes após a construção da Declaração Universal dos Direitos da Criança, em 1989. No Brasil, este fato começou a mudar com a Constituição Federal de 1988 e o Estatuto da Criança e do Adolescente datado 1990, em que ambos proíbem o trabalho infantil. Esses marcos regulatórios autorizam os adolescentes com mais de 14 anos a trabalhem na condição de aprendizes, e que os jovens com idade superior a 16 anos exerçam funções apontadas como seguras (OIT, 2009).

Com base no exposto, o presente estudo teve por objetivo descrever o perfil dos casos de acidentes de trabalho graves em crianças e adolescentes ocorridos entre 2010 a 2018, no estado da Bahia.

\section{MÉTODOS}

Trata-se de um estudo descritivo, de corte transversal, baseado em dados secundários acerca dos casos de acidentes de trabalho grave em crianças e adolescentes, fornecidos pela Diretoria de Vigilância e Atenção à Saúde do Trabalhador (DIVAST). Foram incluídos os acidentes de trabalho graves em crianças e adolescentes notificados ao Sistema de Informações de Agravos de Notificação SINANNET, ocorridos no período compreendido entre os anos de 2010 a 2018, no estado da Bahia. Para definição do acidente de trabalho foi utilizado o código Y 96, da Classificação Internacional do Trabalho (CID 10).
As variáveis analisadas foram divididas em aspectos sociodemográficas e relacionadas ao acidente, sendo as sociodemográficas: sexo, faixa etária, raça/cor, escolaridade, região. As relacionadas ao acidente foram: local do acidente, tipo do acidente, regime de tratamento, ano e evolução do caso.

Os dados foram tabulados e analisados através do Microsoft Office Excel 2010. Para análise dos dados foi realizado cálculo das frequência absolutas e relativas.

Por se tratar de um estudo com base em dados secundários e de domínio público, não houve submissão ao Comitê de Ética em Pesquisa.

\section{RESULTADOS}

No período entre 2010 a 2018 foram identificados 1.525 casos de acidentes de trabalho graves envolvendo crianças e adolescentes, notificados ao SINAN da Bahia. Em 2010 foram registrados 106 casos, aumentando para 211 em 2018, o que significou um acréscimo de quase $100 \%$ no número de AT notificados.

O ano de $2018(n=211)$ obteve o maior quantitativo de acidentes de trabalho notificados, seguido por 2014 $(n=205), 2016(n=199)$. Em contrapartida, os anos que apresentaram menores notificações foram $2011 \quad(n=103)$, $2010(n=106)$ e $2012(n=129)$. Quanto à macrorregião, a que contabilizou maior número de registros foi a leste, (32,3\%; $\mathrm{n}=492)$, seguida da macrorregião sul $(19,7 \%$; $n=301)$. E a que registrou um menor número foi a macrorregião Centro-Norte $(2,2 \% ; n=33)$.

Dando continuidade à análise, verificou-se que grande parte das notificações $(90,7 \% ; n=1383)$ foram do sexo masculino, a maior parte dos acidentados eram adolescentes que estavam na faixa etária de 15 a 19 anos
$(76,2 \% ; \mathrm{n}=1162)$, de raça/cor parda $(51,3 \% ; \mathrm{n}=783)$. É importante destacar um número elevado de acidentes em crianças menores de um ano de idade, o que pode indicar falhas no registro por parte dos profissionais responsáveis.

Quanto à escolaridade predominaram dados ignorados/branco $(37,5 \%$; $\mathrm{n}=572$ ), seguido de não se aplica $(19,1 \% ; n=292)$ e ensino fundamental incompleto $(14,9 \% ; n=227)$ (Tabela 2).

Tabela 1 - Características sociodemográficas das crianças e adolescentes que sofreram acidentes de trabalho graves, na Bahia, no período de 2010 a 2018.

\begin{tabular}{|c|c|c|}
\hline $\begin{array}{c}\text { CARACTERISTICAS } \\
\text { SOCIODEMOGRAFICAS }\end{array}$ & $\mathbf{n}$ & $\%$ \\
\hline \multicolumn{3}{|l|}{ SEXO } \\
\hline Masculino & 1383 & 90,7 \\
\hline Feminino & 142 & 9,3 \\
\hline \multicolumn{3}{|l|}{ FAIXA ETÁRIA } \\
\hline Menor 1 ano & 284 & 19,0 \\
\hline 1 a 4 anos & 2 & 0,0 \\
\hline 5 a 9 anos & 10 & 1,0 \\
\hline 10 a 14 anos & 67 & 4,0 \\
\hline 15 a 17 anos & 440 & 29,0 \\
\hline 18 a 19 anos & 722 & 47,0 \\
\hline \multicolumn{3}{|l|}{ COR/RAÇA } \\
\hline Branca & 157 & 10,3 \\
\hline Preta & 209 & 13,7 \\
\hline Parda & 783 & 51,3 \\
\hline Amarela & 7 & 0,5 \\
\hline Indígena & 3 & 0,2 \\
\hline Sem informação & 366 & 24 \\
\hline \multicolumn{3}{|l|}{ ESCOLARIDADE } \\
\hline Analfabeto & 5 & 0,3 \\
\hline Ensino fundamental incompleto & 320 & 21 \\
\hline Ensino fundamental completo & 56 & 3,7 \\
\hline Ensino médio incompleto & 191 & 12,5 \\
\hline Ensino médio completo & 84 & 5,5 \\
\hline Ensino superior incompleto & 3 & 0,2 \\
\hline Ensino superior completo & 2 & 0,1 \\
\hline Sem informação & 572 & 37,5 \\
\hline Não se aplica & 292 & 19,1 \\
\hline Total & 1525 & 100 \\
\hline
\end{tabular}

Fonte: Sistema de Informações de Agravos de Notificação, 2019

Ao verificar as variáveis que caracterizam o acidente (tabela 2), observou-se que entre as crianças e adolescentes que sofreram acidentes de trabalho graves (ATG), a maioria $(39,4 \% ; n=601)$ estava nas instalações contratantes, seguido por dados ignorados e brancos $(22,2 \% ; n=339)$. O menor registro do local do acidente foi o domicílio $(7,6 \% ; n=116)$. 
Dos ATG notificados, mais da metade foram acidentes típicos $(69,9 \% ; n=1066)$, a maior parte obteve um regime de tratamento hospitalar $(60,8 \% ; n=927)$, dentre as consequências $(33,4 \% ; n=509)$ evoluíram com incapacidade temporária causada pelo ATG e $(2,1 \% ; n=32)$ vieram a óbito.

Tabela 2 - Características do acidente de trabalho grave, segundo local do acidente, tipo do acidente, regime de tratamento e evolução do caso, na Bahia entre os anos de 2010 a 2018.

\begin{tabular}{ccc}
\hline CARACTERISTICAS DO ACIDENTE & \multicolumn{2}{c}{ ACIDENTES } \\
\cline { 3 - 3 } DE TRABALHO GRAVE & $\mathbf{n}$ & $\mathbf{\%}$ \\
\hline LOCAL DO ACIDENTE & & \\
Instalações da contratante & 601 & 39,4 \\
Via pública & 303 & 19,9 \\
Instalações de terceiros & 166 & 10,9 \\
Domicílio próprio & 116 & 7,6 \\
Sem informação & 339 & 22,2 \\
Típico & & \\
Trajeto & 1066 & 69,9 \\
TIPO DE ACIDENTE & 222 & 14,6 \\
Sem informação & 237 & 15,5 \\
Hospitalar & & \\
Ambulatorial & 927 & 60,8 \\
Ambos & 362 & 23,7 \\
Sem informação & 87 & 5,7 \\
REGIME DE TRATAMENTO & 149 & 9,8 \\
Cura & & \\
EVOLUÇÃO DO CASO & 387 & 25,4 \\
Incapacidade Temporária & 509 & 33,4 \\
Incapacidade parcial permanente & 227 & 14,9 \\
Incapacidade total permanente & 13 & 0,9 \\
Óbito pelo acidente & 30 & 2 \\
Óbito por outras causas & 6 & 0,4 \\
Outra & 17 & 1,1 \\
Sem informação & 336 & 22 \\
\hline Total & $\mathbf{1 5 2 5}$ & $\mathbf{1 0 0}$ \\
\hline Sistema de Informações & $\mathrm{A}$ & $\mathrm{Agrav}$ \\
\hline Fonte: &
\end{tabular}

Fonte: Sistema de Informações de Agravos de Notificação, 2019.

\section{DISCUSSÃO}

Crianças e adolescentes acompanham cotidianamente a substituição do seu direito ao lazer, estudo e proteção, pela exploração indiscriminada da sua mão de obra. E por apresentar uma maior fragilidade em relação à população adulta, o público infanto-juvenil está mais propenso a desenvolver incapacidades permanentes ou temporárias, e por vezes evoluem para o óbito.

Em uma década, os casos de acidentes de trabalho graves em crianças e adolescentes no Estado da Bahia obtiveram uma progressão de $99,1 \%$.
Esse dado torna-se alarmante uma vez que tal situação de risco ainda seja negligenciada pelos órgãos governamentais.

Fatores sociais, pessoais e familiares, além do cenário macroeconômico baiano estar representado pelo elevado endividamento das famílias e empresas, associado ao alto nível de desemprego influenciam diretamente para a elevação do índice de trabalho infantil (NASCIMENTO, 2017).

Nesse contexto, a Bahia baseia sua atividade econômica na agropecuária, contudo as especificidades dessa atividade variam de acordo com a microrregião do Estado (NASCIMENTO, 2017). Na presente pesquisa as microrregiões Leste e Sul registraram o maior número de acidentes de trabalho, com base nisso, a partir do conhecimento a respeito das atividades de movimentação financeira de determinada localidade, é possível elaborar planos de ações preventivas e intervencionistas relacionados as atividades laborais direcionados para o público infanto-juvenil que convive em situação de vulnerabilidade para acidentes de trabalho.

Com relação ao sexo das vítimas, os resultados desta pesquisa foram similares aqueles de outras pesquisas nacionais em relação à predominância de indivíduos do sexo masculino (SCUSSIATO et al., 2014; NOVAES; RIBEIRO, 2015). Histórico e culturalmente, a população masculina expõe a maiores fatores de risco, sejam eles para atividade de lazer ou laborais.

Quanto à faixa etária, este estudo constatou que os mais acometidos foram os jovens que se encontravam nas faixas etárias de 18 a 19 e 15 a 17 respectivamente. Tais achados estão em conformidade com os resultados dos estudos de Cavalcante (2015). Em um outro estudo, Silva (2014) evidencia um destaque na faixa etária de 5 a 14 anos, o que entra em discordância com os dados encontrados neste estudo.

Contudo é nítido que durante toda a fase infanto-juvenil há um maior risco de acidente, principalmente pela inexperiência que acompanha esta fase. Neste período da vida os jovens tendem a ter mais disposição para exercer atividades laborais que exigem maior força de trabalho e, muitas vezes, maiores riscos para acidentes (CAVALCANTE, 2015; SILVA; PEREIRA, 2014).

É relevante ressaltar que foram encontradas notificações em crianças menores de um ano de idade. Tal achado suscita a reflexão se os dados foram registrados corretamente no sistema pesquisado.

No presente estudo, $51,3 \%$ dos acidentados estão representados pela cor de pele parda, o que coincidente com outros estudos sobre jovens trabalhadores (PIMENTA, 2013). No entanto, é importante ressaltar que as características demográficas da etnia e cor de pele dependem da multidiversidade cultural e regional do país.

Chama a atenção, na presente investigação, a prevalência dos subregistros no que diz respeito ao nível de instrução do acidentado. Essa lacuna é um dos principais empecilhos em relação à saúde do jovem trabalhador, pois, a falta de informação nas notificações inviabiliza a descrição do perfil sociodemográfico da vítima, bem como, a identificação dos fatoresproblemas e planejamento de propostas intervencionais.

É pertinente ressaltar também, que essas limitações de informações resultam no desconhecimento acerca do impacto do trabalho sobre a saúde das crianças e adolescentes que atuam 
no mercado formal e informal. No Rio Grande do Norte, a ocorrência de acidentes de trabalho grave, verificada através do Sistema de Informação de Agravos de Notificação, apresentou subregistros em algumas variáveis importantes acerca do agravo (CAVALCANTE et al., 2015).

Apesar da variável a respeito da escolaridade apresentar alto índice de subregistros, os resultados apontam o ensino fundamental incompleto como maior de grau de instrução dos trabalhadores acidentados. Uma pesquisa realizada em um Estado do nordeste brasileiro mostrou que o risco de sofrer um acidente de trabalho é cinco vezes maior em pessoas com analfabetismo ou baixa escolaridade (CAVALCANTE et al., 2015). Ademais, aponta-se que, ao se tratar de crianças e adolescentes, espera-se um percentual de escolaridade nessa faixa de escolaridade.

A maior proporção dos acidentes de trabalho sucedeu-se nas instalações do contratante, sendo descrito como típico, ou seja, ocorreu no próprio ambiente laboral. $\mathrm{O}$ que pode ser justificado pela carga horária de trabalho exacerbada que essas crianças e adolescentes estão submetidos.

O menor registro do local do acidente foi o domicilio, justamente pelo fato de que a demanda de trabalho para esse público é maior fora dele, nas dependências do empregador.

Sobre os tipos de acidentes os típicos foram responsáveis por mais da metade dos casos, corroborando com o estudo desenvolvido por Pimenta e colaboradores no qual $76,9 \%$ dos casos de acidentes de trabalho foram típicos, seguidos pelos de trajeto que correspondeu a 23,1 \% (PIMENTA et al., 2013).

A maior parte dos acidentados obteve tratamento hospitalar. Aponta- se que a grande parte dos acidentes graves necessita de atendimento especializado. Cavalcante e colaboradores ao realizar um estudo em ambiente hospitalar encontraram que os AT são responsáveis por $25 \%$ das lesões por causas externas recebidas nos serviços de emergência que incluem atendimentos a adultos e jovens (CAVALCANTE et al., 2015).

$\mathrm{E}$, dentre as consequências dos AT, 33,4\% evoluíram com incapacidade temporária em decorrência do AT, corroborando a uma pesquisa realizada na cidade de Ipatinga que evidenciou que $14,9 \%$ dos adolescentes acometidos tiveram de se afastar das atividades laborais sendo (SANTOS et al., 2009). Este percentual mais elevado pode ser justificado pelo aumento da entrada dos adolescentes no mercado de trabalho, devido a situação econômica enfrentada no Brasil nos últimos anos.

Com base no exposto, fazem-se necessárias mobilizações regionais, nacionais e internacionais acerca da garantia dos direitos das crianças e adolescentes, e também da importância que o papel da educação como uma das ferramentas mais eficazes de combate ao trabalho infantil.

\section{CONCLUSÃO}

O trabalho infanto-juvenil é um fato preocupante e crescente, tanto em âmbito nacional quanto internacional, por tratar-se de um público de alta vulnerabilidade, exposto a situações insalubres de trabalho e a exploração da mão de obra, que compromete a saúde, o desenvolvimento, rendimento escolar e os relacionamentos no âmbito familiar e social dessas crianças e jovens.

O presente estudo aponta que, a maioria dos casos de acidentes de tra- balho graves ocorre no ambiente de trabalho das crianças e adolescentes e que a predominância dos sub-registros está relacionada ao nível de escolaridade desses trabalhadores. Fatores sociais, familiares e as atividades desenvolvidas em cada localidade do estado influenciam a entrada cada vez mais precoce no mercado de trabalho e o tipo de acidente com maior predominância.

Diante do que foi tratado, evidencia-se a importância das esferas governamentais e demais órgãos competentes tanto na fiscalização dos locais de trabalho, assegurando que esses estejam em condições ideais de execução das atividades laborais, quanto no desenvolvimento de políticas públicas que informam e previnam acidentes e que amparem as crianças e adolescentes acidentados.

\section{REFERÊNCIAS}

BRASIL. Ministério da Saúde (BR). Notificação de acidentes de trabalho fatais, graves e com crianças e adolescentes. Brasília (DF): 2006.

CAVAlCANTE, C. A. A. et al. Acidentes de trabalho grave no Rio Grande do Norte: estudo transversal. Online BrazilianJournalOfNursing, v. 14, p. 543-55, 2015.

NASCIMENTO, C.; BRITTO, E.; SANTANA, P.M. Economia baiana: retrospectiva 2017 e perspectivas.

Bahia anál. dados, v. 27, n. 2, p. 31 53, jul.-dez, 2017

NOVAES, D. G.; RIBEIRO, L. A. O. Perfil epidemiológico dos acidentes de trabalho graves notificados do ano de 2011 a novembro de 2014 no município de Araguatins-To, Brasil. Humanidades e inovação, Palmas, v. 2, n. 2, p. 6-17, 2015.

OIT. Organização Internacional do Trabalho. Boas práticas do setor 
saúde para a erradicação do traba-

lho infantil. Brasília (DF): OIT, 2009.

PIMENTA, A. A. et al. Acidentes de trabalho ocorridos entre adolescentes. Texto Contexto Enferm. Florianópolis, v. 22, n. 2, p. 279-84, 2013.

SANTOS, M. E. A. et al. Trabalho Precoce e Acidentes Ocupacionais na Adolescência Esc Anna Nery RevEnfer ,v. 13, n. 4, p. 824-32, 2009.

SCUSSIATO, L. A. et al. Perfil epidemiológico dos acidentes de trabalho graves no Estado do Paraná, Brasil, 2007 a 2010. Epidemiol. Serv. Saúde, Brasília, v. 22, n. 4, p. 621-630, 2013.

SILVA, A. C. C.; PEREIRA, T. C. L. Perfil e custos das internações hospitalares por acidentes de trabalho na região sudoeste da Bahia no período de 2005 a 2007. RevBrasEpidemiol, v. 17, n. 2, p. 381-394, 2014.

SILVEIRA, R. C. P.; ROBAZZI, M. L. C. C.Estilo de vida e trabalho de crianças e adolescentes em Programas Saúde da Família. Rev. Eletr. Enf. [Internet], v. 19, n. 10, p. 1-11, 2017. 